\section{SP0160 IS DIABETES AN INFLAMMATORY DISEASE AND SHOULD BE TREATED LIKE THAT?}

A. Schaeffler. Department of Internal Medicine-III, Endocrinology and Diabetes, University of Giessen, Germany, Giessen, Germany

It is a well-known clinical observation that inflammatory diseases are accompanied by metabolic implications such as hyperglycemia, insulin resistance and increased fatty acids. On the other hand, metabolic diseases have inflammatory implications. There is a chronic and low-grade state of inflammation in obesity and type 2 diabetes. Physiological insulin resistance during infection or inflammation redistributes glucose and fatty acids to immune cells. On the basis of systemic and adipose tissue inflammation such as MCP-1/TLR-4-driven macrophage infiltration and pro-inflammatory polarization of these cells, this lecture will give a summary of anti-diabetic effects of anti-inflammatory drugs used in rheumatology.

What is the evidence from clinical studies using anti-inflammatory approaches to treat patients with type 2 diabetes mellitus?

Basically, TNF plays an important role in insulin resistance in rodents. Blocking TNF in rodents reverses obesity-related diabetes. There are no state of the art clinical studies showing convincing evidence of an anti-diabetic potential due to underpowered cohorts and short duration of the studies. Only one single study over 6 months showed a 10\% improvement of fasting glucose levels in 40 prediabetic obese patients.

Diacerein belongs to the chemical group of anthranoids. Although the mechanism of action is unknown, diacerein decreases the levels of TNF and of IL1b and has therefore been used in rheumatic diseases. Diacerein has potent effects on insulin secretion and glycemic control with a reduction of HBA1c level by $1.6 \%$. IL-1 receptor antagonists such as anakinra (a recombinant human IL-1 receptor antagonist) have also been studied in diabetes. Anakinra was able to improve glycemia, inflammation and insulin secretion. Two additional studies in patients with impaired glucose tolerance or prediabetes demonstrated positive effects of anakinra on beta cell secretory capacity. Since anakinra requires daily injections and often causes adverse effects at the injection site, humanized antibodies against IL $1 \mathrm{~b}$ have been developed. Each of these antibodies had beneficial effects in patients with type 2 diabetes. However, studies were either underpowered or showed only little improvements of glycosylated HBA1c levels, probably due to low pre-study levels.

Inhibition of the IKK/NFkB pathway might also be of benefit in insulin resistance. Salsalate improves insulin sensitivity, insulin secretion and glycemic control to a moderate extent of $0.4-0.5 \%$. Salsalate also has effects in prediabetic patients and in drug-naive type 2 diabetic patients.

There is upcoming evidence of potential benefical effects of CCR2 antagonism in diabetes.

Obesity causes an increased flux of fatty acids into muscle and liver with an accumulation of DAG in these organs. DAG activates specific isoforms of protein kinases, that is PKC T in muscle and PKC E in the liver. These isoforms cause an inhibitory phosphorylation of IRS-1 and the insulin receptor itself leading to insulin resistance. Weight reduction by diet, physical activity and bariatric surgery remove ectopic fat and are able reverse diabetes. Most interestingly, PKC-isoform specific inhibitors such as FGF-21 might represent new drug targets.

Finally, targeting ectopic fat is more close to the pathophysiology of diabetes than anti-inflammatory therapy.

Disclosure of Interest: None declared

DOI: 10.1136/annrheumdis-2017-eular.7055

\section{SP0161 THE ROLE OF ADIPOSE TISSUE IN OSTEOARTHRITIS}

\section{A. Ioan-Facsinay. Leiden University Medical Center, Leiden, Netherlands}

The association between obesity and osteoarthritis $(\mathrm{OA})$ is nowadays wellaccepted. This association is present both in weight-bearing and non-weightbearing joints, indicating that next to local effects, systemic effects associated with obesity could play a role in disease. The relative contribution of local and systemic effects could be different in each joint. In this presentation, I will summarize the current knowledge about the contribution of local and systemic adipose tissues to osteoarthritis in different joints, with emphasis on knee OA. Moreover, obesity-associated changes and their role in OA will be highlighted.

The key messages I would like to convey are:

- Mechanical stress is a major contributor to knee OA

- The infrapatellar fat pad (IFP), an intra-articular adipose tissue in the knee, is dostinct from other adipose tissues and could play a role in knee $O A$

- Only few obesity-related features have been described in IFP in OA

Disclosure of Interest: None declared

DOI: 10.1136/annrheumdis-2017-eular.7207

\section{SP0162 ADIPOKINES IN THE PATHOPHYSIOLOGY OF CARTILAGE}

O. Gualillo. NEIRID Lab (Laboratory of NeuroEndocrine Interactions In Rheumatology and Inflammatory Diseases), Sergas Idis, Santiago de Compostela, Spain

Fat mass dysregulation is a marked characteristic of overweight and obesity. Obesity is, historically, a classic risk factor for osteoarthritis development and progression and shares with osteoarthritis a huge societal impact but also several common biochemical features that are strongly related to the low-grade inflammatory status. Even though OA has been considered during decades a disabling degenerative disease related to mechanical, age and genetic factors with poor inflammatory component, the current consensus is that OA, in particular $\mathrm{OA}$ associated with metabolic alterations, is a global muscle-skeletal diseases in which pro-inflammatory and catabolic mediators, most of them produced by a dysregulated visceral or periarticular white adipose tissue (WAT), are at play. The coexistence of obesity and $\mathrm{OA}$ is a clinical reality and is changing the traditional view of this degenerative disorder. Thus, chronic low-grade inflammation is a common characteristic shared by both obesity and OA. It has been demonstrated that obesity increases the incidence of OA, particularly in weight-bearing joints such as knees. However, the fact that obese individuals have an increased risk of developing $\mathrm{OA}$ in non-weight bearing joints such as hands and wrists suggests that factors produced by WAT play a role in the onset and/or progression of OA. In addition to a growing body of evidence demonstrating that obesity has a direct mechanical effect joint cartilage, recent research shows that pro-inflammatory factors produced by WAT (collectively known as adipokines) promote further inflammation and degradation of cartilage, also influencing the whole joint environment (i.e. synovium, muscle, bone and immune cells). Accumulating evidence shows another potential source of inflammatory adipokines in the joint is the Hoffa infrapatellar fat pad. Adipokines including leptin adiponectin, visfatin and lipocalin 2 have been demonstrated to exhibit a wide spectrum of biological activity including the activation of pro-inflammatory and catabolic pathways mediated by elevated levels of NO, ROS, MMPs and PGE2. The discovery of adipokines has made a major contribution to our understanding of the complex relationship between diabesity and $\mathrm{OA}$, encompassing a variety of factors that include the immune system, metabolism and biomechanics. A critical aspect that must constantly be borne in mind is that diabesity and $\mathrm{OA}$ share a low-grade inflammatory state that heavily influences the course of OA progression. Therefore, the prevention and correction of diabesity should be the first line approach for tackling the detrimental effects of weight gain, adiposity and altered metabolic WAT function in OA. The aim of this lecture is to summarize the role of adipokines in bone and cartilage function, as well as in inflammatory and degenerative joint disease. We discuss clinical implications and then survey attempts to exploit this role for therapeutic gain, which holds potential as a novel approach for drug development in bone and joint disease.

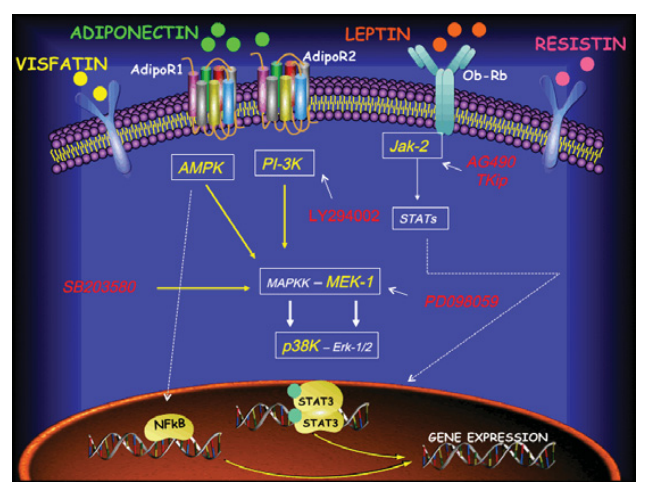

Disclosure of Interest: None declared

DOI: 10.1136/annrheumdis-2017-eular.7199

\section{SATURDAY, 17 JUNE 2017 \\ Trials and tribulations of medication adherence}

\section{SP0163 INTERVENTIONS TO IMPROVE MEDICATION ADHERENCE IN PATIENTS WITH INFLAMMATORY ARTHRITIS}

\section{J.E. Vriezekolk. Rheumatology, Sint Maartenskliniek, Nijmegen, Netherlands}

Non-adherence to medication in rheumatic diseases is a major concern for patient's health, because incorrect use can result in less therapeutic benefits of treatment, including more disease activity and increased radiological damage. As a consequence, non-adherence may lead to more disability and lower healthrelated quality of life. Using Lowe's taxonomy ${ }^{1}$, the current state of evidence around medication adherence interventions will be presented. Examples of promising intervention strategies will be highlighted.

References:

[1] Lowe et al. (2011) Development of a taxonomy of interventions to organise the evidence on consumers' medicine use.

Disclosure of Interest: None declared

DOI: 10.1136/annrheumdis-2017-eular.7081 